Journal of Mathematics and Statistics 7 (1): 68-72, 2011

ISSN 1549-3644

(C) 2010 Science Publications

\title{
A Note on the Numerical Solution for Fredholm Integral Equation of the Second Kind with Cauchy kernel
}

\author{
M. Abdulkawi, N.M.A. Nik Long and Z.K. Eshkuvatov \\ Department of Mathematics and Institute for Mathematical Research, \\ University Putra Malaysia, 43400 Serdang, Selangor, Malaysia
}

\begin{abstract}
Problem statement: In this study, numerical solution for the Fredholm integral equation of the second kind with Cauchy singular kernel is presented. Approach: The Chebyshev polynomials of the second kind are used to approximate the unknown function. Results: Numerical results are given to show the accuracy of the present numerical solution. Conclusion: The present numerical solution to the Fredholm integral equation of the second kind with Cauchy kernel is accurate.
\end{abstract}

Key words: Integral equations, Cauchy singular kernel, Chebyshev polynomial approximations

\section{INTRODUCTION}

Consider the Fredholm integral equation of the second kind with Cauchy kernel of the form:

$\varphi(x)+\lambda \int_{-1}^{1} \frac{\varphi(t)}{t-x} d t=f(x),(\lambda$ is a constant $)$

with specified end conditions:

$\varphi( \pm 1)=0$

where $\mathrm{f}$ is assumed to be real-valued functions belong to the class of Holder on the interval $[-1,1]$ and $\varphi$ is the unknown function to be determined. The singular integral in Eq. 1 being understood in the sense of Cauchy principal value. Many researchers devoted their works on approximating the singular integrals of Cauchy type. Particularly, Dagnino and Santi (1990) obtained a product quadrature rules, based on spline interpolation, for the numerical evaluation of Cauchy singular integrals. They also proposed an error bound and obtained convergence results for functions $\mathrm{f} \in \mathrm{C}^{\mathrm{k}}[-$ $1,1], \mathrm{k}=1,2$ or 3 . Orsi (1990) proved the uniform convergence of some quadrature formulas based on spline approximation for Cauchy principal value integral. They also presented some numerical applications. In particular, they applied their rules to the well-known Prandtl's integral equation. Rabinowitz (1990) proved convergence results for product integration rules based on approximating splines. These results are both for bounded and unbounded integrands.
Pointwise and uniform convergence results are proved for sequences of Cauchy principle values of these approximating splies. .Dagnino and Santi (1991) considered the same rules as in Dagnino and Santi (1990) and investigated their convergence for a large class of functions $\mathrm{f}$. They established an error bound and some uniform convergence results in the case of equally spaced quadrature nodes, for function $\mathrm{f}$, satisfying a Holder condition of order $\mu$ on $[-1,1], 0<\mu \leq 1$. Hasegawa and Torii (1991) presented an automatic quadrature for computing Cauchy principal value integrals for smooth functions $\mathrm{f}(\mathrm{t})$. They approximated the function $f(t)$ by a sum of Chebyshev polynomials whose coefficients are computed using the Fast Fourier Transform (FFT). Hasegawa and Torii (1994) presented an automatic quadrature for approximating Hadamard finite-part (fp) integrals of a smooth function, with a double pole singularity within the range of integration. The quadrature rule is drived from the differentiation of an approximation to a Cauchy principal value integral. Diethelm (1995) investigated the numerical approximation of the Cauchy principal value integral. He presented the quadrature formula for approximating the Cauchy principal value integral. He proved the convergence of the quadrature formula and gave the estimation for the errors. Dagnino and Lamberti (1996) evaluated the Cauchy principal value integral by applying a local spline approximation method, defined for any function $\mathrm{f} \in \mathrm{L}_{1}[-1,1]$. They established convergence results with error bound. Diethelm (1997) considered the so-called modified quadrature formulas, i.e. formulas obtained by first subtracting out the singularity and then applying a classical quadrature

Corresponding Author: Dr. Mohammad Abdulkawi, Department of Mathematics and Institute for Mathematical Research, University Putra Malaysia, 43400 Serdang, Selangor, Malaysia 
formula, for the numerical approximation of Cauchy principal value integrals. They have given new bounds involving the total variation $\operatorname{Var} \mathrm{f}^{(\mathrm{s})}$ and $\mathrm{L}_{\mathrm{p}}$-norms $\left\|\mathrm{f}^{(\mathrm{s})}\right\|_{\mathrm{p}}$ of some derivative of the integrand function. Eshkuvatov et al. (2009) constructed a new quadrature formulas for evaluating the singular integral of Cauchy type. The construction of the quadrature formulas is based on the modification of discrete vortices method and linear spline interpolation over the finite interval $[-1,1]$. They proved that the constructed quadrature formula converges for any singular point $\mathrm{x}$ not coinciding with the end points of the interval $[1,1]$. They have given error bounds in the classes of functions $\mathrm{H}^{\alpha}[-1,1]$ and $\mathrm{C}^{1}[-1,1]$ of order $\mathrm{O}\left(\mathrm{h}^{\alpha}|\ln \mathrm{h}|\right), 0<\alpha \leq 1$ and $\mathrm{O}(\mathrm{h}|\ln \mathrm{h}|)$, respectively. Eshkuvatov et al. (2010) considered the singular integral with the Cauchy kernel. They constructed new quadrature formulas based on the modification of discrete vortex method to approximate the singular integral. They have shown error bounds in the classes of functions $\mathrm{H}^{\alpha}[-1,1]$ and $\mathrm{C}^{1}[-1,1]$ for either $x=t_{j}$ or $x=t_{0 j}$ where $t_{j}, j=1,2, \ldots, N$ are the node points and $t_{0 j}=\left(t_{j}+t_{j+1}\right) / 2$.

The integral equations with Cauchy kernel have been widely used in solving problems associated with aerodynamic, hydrodynamic and elasticity (Lifanov, 1996; Ladopoulos, 2000; Abdou and Naser, 2003; Mohankumar and Natarajan, 2008; Lara and Mariagrazia, 2005; Kasozi and Paulsen, 2005a; Kasozi and Paulsen, 2005b; Ganji et al., 2008; Thukral, 2005).

In this study, we present a numerical solution for the Eq. 1 with conditions (2).

\section{MATERIALS AND METHODS}

The unknown function $\varphi$ of Eq. 1 which satisfies conditions (2) can be represented as:

$$
\varphi(x)=\sqrt{1-x^{2}} \psi(x), \quad-1 \leq x \leq 1
$$

where, $\psi(\mathrm{x})$ is a well behaved function of $\mathrm{x}$ on the interval $[-1,1]$. The function $\psi(x)$ in Eq. 3 is approximated using the Chebyshev polynomials of the second kind, $\mathrm{U}_{\mathrm{i}}$, as:

$$
\psi_{\mathrm{i}}(\mathrm{x}) \approx \sum_{\mathrm{i}=0}^{\mathrm{N}} \mathrm{a}_{\mathrm{i}} \mathrm{U}_{\mathrm{i}}(\mathrm{x})
$$

where:

$$
\mathrm{U}_{\mathrm{i}-1}(\mathrm{x})=\frac{\sin \left[\mathrm{i} \cos ^{-1}(\mathrm{x})\right]}{\sin \left[\cos ^{-1}(\mathrm{x})\right]}, \quad \mathrm{i}=0,1, \ldots, \mathrm{N}
$$

From (3) and (4), we have:

$$
\varphi(\mathrm{x}) \approx \sqrt{1-\mathrm{x}^{2}} \sum_{\mathrm{i}=0}^{\mathrm{N}} \mathrm{a}_{\mathrm{i}} \mathrm{U}_{\mathrm{i}}(\mathrm{x}), \quad-1 \leq \mathrm{x} \leq 1
$$

Substituting (6) into (1), yields:

$$
\begin{aligned}
& \sqrt{1-\mathrm{x}^{2}} \sum_{\mathrm{i}=0}^{\mathrm{N}} \mathrm{a}_{\mathrm{i}} \mathrm{U}_{\mathrm{i}}(\mathrm{x}) \\
& +\lambda \sum_{\mathrm{i}=0}^{\mathrm{N}} \mathrm{a}_{\mathrm{i}} \int_{-1}^{1} \frac{\sqrt{1-\mathrm{t}^{2}} \mathrm{U}_{\mathrm{i}}(\mathrm{t})}{\mathrm{t}-\mathrm{x}} \mathrm{dt}=\mathrm{f}(\mathrm{x})
\end{aligned}
$$

It is known that (Kythe and Schaferkotter, 2005; Abdulkawi et al., 2009)

$\int_{-1}^{1} \frac{\sqrt{1-t^{2}} U_{i}(t)}{t-x} d t=-\pi T_{i+1}(x)$

where, $T_{i}$ are the Chebyshev polynomials of the first kind which is defined by:

$\mathrm{T}_{\mathrm{i}}(\mathrm{x})=\cos \left[\mathrm{i} \cos ^{-1}(\mathrm{x})\right], \quad \mathrm{i}=0,1, \ldots, \mathrm{N}$

Using (8) into Eq. 7, yields:

$$
\sum_{i=0}^{N} a_{i}\left[\sqrt{1-x^{2}} U_{i}(x)-\pi \lambda T_{i+1}(x)\right]=f(x)
$$

Multiplying both sides of Eq. 10 by $\mathrm{U}_{\mathrm{i}}$ and integrating from -1 to 1 , we obtain the following system of linear equations;

$\sum_{i=0}^{N} a_{i} A_{i j}=d_{j}, \quad j=0,1, \ldots, N$

where:

$$
\begin{aligned}
& A_{i j}=B_{i j}+C_{i j} \\
& B_{i j}=\int_{-1}^{1} \sqrt{1-t^{2}} U_{i}(t) U_{j}(t) d t= \begin{cases}\frac{\pi}{2}, & i=j \\
0, & i \neq j\end{cases} \\
& C_{i j}=-\pi \lambda \int_{-1}^{1} T_{i+1}(t) U_{j}(t) d t
\end{aligned}
$$

and:

$$
d_{j}=\int_{-1}^{1} f(t) U_{j}(t) d t
$$


Solving the above system for the unknown coefficients $a_{i}, i=0,1, \ldots, N$ and substituting the values of $a_{i}$ into the approximate solution (6), we obtain the numerical solution of the Eq. 1 with conditions (2).

\section{RESULTS AND DISCUSSION}

Example 1: Let us consider the singular integral equation:

$\varphi(x)+\int_{-1}^{1} \frac{\varphi(t)}{t-x} d t=-2 \pi x^{2}+\left(2 \sqrt{1-x^{2}}-\pi\right) x+\sqrt{1-x^{2}}+\pi(16)$

with the conditions:

$\varphi( \pm 1)=0$

It is not difficult to see that the exact solution of the Eq.16 is:

$$
\phi(x)=\sqrt{1-x^{2}}(2 x+1)
$$

Due to Eq. 12-14, we obtain:

$$
\begin{aligned}
& \mathrm{A}_{00}=\frac{\pi}{2}, \quad \mathrm{~A}_{10}=\frac{2}{3} \pi, \quad \mathrm{A}_{20}=0, \\
& \mathrm{~A}_{01}=-\frac{4}{3} \pi, \quad \mathrm{A}_{11}=\frac{\pi}{2}, \quad \mathrm{~A}_{21}=\frac{4}{5} \pi \\
& \mathrm{A}_{02}=0, \quad \mathrm{~A}_{12}=-\frac{6}{5} \pi, \quad \mathrm{A}_{22}=\frac{\pi}{2} \text {. }
\end{aligned}
$$

From Eq. (15), we have:

$$
\begin{aligned}
d_{j}= & \int_{-1}^{1}\left(-2 \pi t^{2}+\left(2 \sqrt{1-t^{2}}-\pi\right) t\right) U_{j}(t) d t \\
& +\int_{-1}^{1}\left(\sqrt{1-t^{2}}+\pi\right) U_{j}(t) d t
\end{aligned}
$$

which gives:

$$
\begin{aligned}
& \mathrm{d}_{0}=\frac{2}{3} \pi+\frac{1}{2} \pi, \\
& \mathrm{d}_{1}=-\frac{4}{3} \pi+\frac{1}{2} \pi, \\
& \mathrm{d}_{2}=-\frac{6}{5} \pi
\end{aligned}
$$
becomes:

Thus the system of linear equations (11) for $\mathrm{N}=2$

$$
\begin{aligned}
& \mathrm{a}_{0}+\frac{2}{3} \pi \mathrm{a}_{1}=\frac{2}{3} \pi+\frac{\pi}{2}, \\
& -\frac{4}{3} \pi \mathrm{a}_{0}+\mathrm{a}_{1}+\frac{4}{5} \pi \mathrm{a}_{2}=-\frac{4}{3} \pi+\frac{\pi}{2}, \\
& -\frac{6}{5} \pi \mathrm{a}_{1}+\mathrm{a}_{2}=-\frac{6}{5} \pi .
\end{aligned}
$$

It is easy to see that the solution to the above system is:

$$
a_{0}=1, \quad a_{1}=1, \quad a_{2}=0
$$

Substituting (23) into (6) for $\mathrm{N}=2$, we obtain:

$$
\varphi(\mathrm{x})=\sqrt{1-\mathrm{x}^{2}}(1+2 \mathrm{x})
$$

which is the exact solution.

Example 2: Consider the following equation:

$\varphi(x)+\int_{-1}^{1} \frac{\varphi(t)}{t-x} d t=\sqrt{1-x^{2}} U_{5}(x)-\pi T_{6}(x)$

with the conditions:

$\varphi( \pm 1)=0$

It is clear that the exact solution of the Eq. 25 is:

$\varphi(x)=\sqrt{1-x^{2}}\left(32 x^{5}-32 x^{3}+6 x\right)$

From Eq. 12-14, we obtain:

$$
\begin{aligned}
& \mathrm{A}_{00}=2, \mathrm{~A}_{10}=0, \mathrm{~A}_{20}=\frac{2}{3}, \mathrm{~A}_{30}=0, \\
& \mathrm{~A}_{40}=\frac{2}{5}, \mathrm{~A}_{50}=0, \mathrm{~A}_{01}=-\frac{\pi^{2}}{2}, \mathrm{~A}_{11}=\frac{4}{3}, \\
& \mathrm{~A}_{21}=0, \mathrm{~A}_{31}=\frac{8}{15}, \mathrm{~A}_{41}=0, \mathrm{~A}_{51}=\frac{12}{35}, \\
& \mathrm{~A}_{02}=-\frac{2}{3}, \mathrm{~A}_{12}=-\frac{\pi^{2}}{2}, \mathrm{~A}_{22}=\frac{6}{5}, \mathrm{~A}_{32}=0, \\
& \mathrm{~A}_{42}=\frac{10}{21}, \mathrm{~A}_{52}=0, \mathrm{~A}_{03}=0, \mathrm{~A}_{13}=-\frac{4}{5}, \\
& \mathrm{~A}_{23}=-\frac{\pi^{2}}{2}, \mathrm{~A}_{33}=\frac{8}{7}, \mathrm{~A}_{43}=0, \mathrm{~A}_{53}=\frac{4}{9}, \\
& \mathrm{~A}_{04}=\frac{-2}{15}, \mathrm{~A}_{14}=0, \mathrm{~A}_{24}=\frac{-6}{7}, \mathrm{~A}_{34}=\frac{-\pi^{2}}{2}, \\
& \mathrm{~A}_{44}=\frac{10}{9}, \mathrm{~A}_{54}=0, \mathrm{~A}_{05}=0, \mathrm{~A}_{15}=-\frac{4}{21}, \\
& \mathrm{~A}_{25}=0, \mathrm{~A}_{35}=-\frac{8}{9}, \mathrm{~A}_{45}=\frac{\pi^{2}}{2}, \mathrm{~A}_{55}=\frac{12}{11}
\end{aligned}
$$


Due to Eq. 15, we have:

$d_{j}=\int_{-1}^{1}\left(\sqrt{1-t^{2}} U_{5}(t)-\pi T_{6}(t)\right) U_{j}(t) d t$

which gives:

$$
\begin{aligned}
& \mathrm{d}_{0}=0, \quad \mathrm{~d}_{1}=\frac{12}{35}, \quad \mathrm{~d}_{2}=0, \\
& \left.\mathrm{~d}_{3}=\frac{4}{9}, \quad \mathrm{~d}_{4}=0, \quad \mathrm{~d}_{5}=\frac{12}{11} . \quad\right\}
\end{aligned}
$$

Thus the corresponding system of linear equations for $\mathrm{N}=5$ is:

$$
\sum_{i=0}^{5} a_{i} A_{i j}=d_{j}, \quad j=0,1, \ldots, 5
$$

where, $A_{i j}, i, j=0,1, \ldots, 5$ are defined in $(28)$ and $d_{j}, j=$ $0,1, \ldots, 5$ are defined in (30).

It is not difficult to see that the solution to the above system is:

$$
\mathrm{a}_{0}=0, \mathrm{a}_{1}=0, \mathrm{a}_{2}=0, \mathrm{a}_{3}=0, \mathrm{a}_{4}=0, \mathrm{a}_{5}=1
$$

Substituting the values of $a_{i}, i=0,1, \ldots, 5$ into (6) where $\mathrm{N}=5$, we obtain the numerical solution of Eq. 25 which is identical to the exact solution (27).

\section{CONCLUSION}

The truncated series involving the Chebyshev polynomial approximation of the second kind is used to approximate the unknown function for solving the Fredholm integral equation of the second kind with Cauchy kernel and constant coefficients. The method of approximation illustrated here gives a good way for obtaining the numerical solution avoiding the complicated integrations. Numerical results show the accuracy of the method presented which, for some functions $\mathrm{f}(\mathrm{x})$, gives the exact solution.

\section{ACKNOWLEDGEMENT}

This study was supported by University Putra Malaysia under Postdoctoral Fellowship and project No. 05-03-10-0988RU.

\section{REFERENCES}

Abdou, M., A. and A.A. Naser, 2003. On the numerical treatment of the singular integral equation of the second kind. Applied Math. Computation, 146: 373-380. DOI: 10.1016/S0096-3003(02)00587-8
Abdulkawi, M., Z.K. Eshkuvatov and N.M.A.N. Long, 2009. A numerical treatment for solving Cauchy type singular integral equation. FJAM., 35: 171-180.

Dagnino, C. and E. Santi, 1990. Spline product quadrature rules for cauchy singular integrals. J. Comput. Applied Math., 33: 133-140. DOI: 10.1016/0377-0427(90)90363-5

Dagnino, C. and E. Santi, 1991. On the convergence of spline product quadratures for Cauchy principal value integrals. J. Comput. Applied Math., 36: 181-187. DOI: $10.1016 / 0377-0427(91) 90025-\mathrm{F}$

Dagnino, C. and P. Lamberti, 1996. Numerical evaluation of Cauchy principal value integrals based on local spline approximation operators. J. Comput. Applied Math., 76: 231-238. DOI: 10.1016/S0377-0427(96)00105-7

Diethelm, K. , 1995. Gaussian quadrature formulae of the third kind for Cauchy principal value integrals: Basic properties and error estimates. J. Comput. Applied Math., 65: 97-114. DOI: 10.1016/03770427(95)00103-4

Diethelm, K., 1997. New error bounds for modified quadrature formulas for Cauchy principal value integrals. J. Comput. Applied Mathe., 82: 93-104. DOI: $10.1016 / \mathrm{S} 0377-0427(97) 00045-9$

Eshkuvatov, Z.K., N.M.A.N. Long and M. Abdulkawi, 2009. Quadrature formula for approximating the singular integral of Cauchy type with unbounded weight function on the edges. J. Comput. Applied Math., 233: 334-345. 10.1016/j.cam.2009.07.034

Eshkuvatov, Z.K., N.M.A.N. Long and M. Abdulkawi, 2010. Numerical evaluation for Cauchy type singular integrals on the interval. J. Comput. Applied Math., 233: 1995-2001. DOI: 10.1016/j.cam.2009.09.034

Ganji, D.D., M. Amini and A. Kolahdooz, 2008. Analytical investigation of Hyperbolic equations via he's methods. Am. J. Eng. Applied Sci., 1: 399-407. DOI: 10.3844/ajeassp.2008.399.407

Hasegawa, T. and T. Torii, 1991. An automatic quadrature for Cauchy principal value integrals. Mathe. Comput., 56: 741-54.

Hasegawa, T. and T. Torii, 1994. Hilbert and hadamard transforms by generalized chebyshev expansion. J. Comput. Applied Math., 51: 71-83. DOI: 10.1016/0377-0427(92)00003-R

Kasozi, J. and J. Paulsen, 2005a. Flow of dividends under a constant force of interest. Am. J. Applied Sci., 2: 1389-1394. DOI: 10.3844/ajassp.2005. 1389.1394

Kasozi, J. and J. Paulsen, 2005b. Numerical ultimate ruin probabilities under interest force. J. Math. Stat., $\quad$ 1: 246-251. DOI: 10.3844/jmssp.2005.246.251 
Kythe, K.P. and R.M. Schaferkotter, 2005. Hand-book of computational Methods for integration. 1st Edn., Chapman and Hall /CRC Press, London, ISBN-10: 1584884282, pp: 598.

Ladopoulos, E., 2000. Singular integral equations: Linear and Non-Linear Theory and its applications in Science and Engineering. 1st Edn., Springer Verlag, USA., ISBN-10: 9783540672302 , pp: 584.

Lara, F. and G. Mariagrazia, 2005. Parsimonious var models for air pollution dynamic analysis. J. Math. Stat., 1:258-267. 10.3844/jmssp.2005.258.267

Lifanov, I. K., 1996. Singular Integral Equation and Discrete Vortices. 1st Edn., Walter de Gruyter, Croatia, ISBN-10: 906764207X, pp: 475.
Mohankumar, N. and A. Natarajan, 2008. On the numerical solution of cauchy singular integral equations in neutron transport. Annals Nuclear Energy, 35 : $1800-1804$ DOI: 10.1016/j.anucene.2008.05.007

Orsi, A.P., 1990. Spline approximation for Cauchy principal value integrals. J. Comput. Applied Math., 30: 191-201. DOI: 10.1016/0377-0427(90)90027-W

Rabinowitz, P., 1990. Numerical integration based on approximating splines. J. Comput. Applied Math., 33: 73-83. DOI: 10.1016/0377-0427(90)90257-Z

Thukral, R., 2005. Introduction to the new type of algorithms for accelerating convergence of sequence. J. Math. Stat., 1: 15-23. DOI: 10.3844/jmssp.2005.15.23 